\title{
Team learning in teacher teams: Team entitativity as a bridge between teams-in- theory and teams-in-practice
}

Katrien Vangrieken, Filip Dochy, \& Elisabeth Raes

Although teams are increasingly prevalent in practice and research on teams and team learning is constantly rising, current research appears to focus on 'real teams' that meet certain strict and pre-defined criteria. However, not all teams in all contexts appear to meet these criteria. Teacher teams for example are often not real teams as defined in contemporary team literature and thus this context is underexplored in research on team learning. This paper focuses on these ambiguous teams. It uses the term 'team entitativity' to describe this ambiguity and to present the team concept as a continuum ranging from a mere aggregate of individuals to a real team. This makes it possible to apply 'team learning' frameworks to teams that do not strictly meet predefined team criteria. As such, we investigated the Team Learning Beliefs \& Behaviours model to teacher teams in colleges for higher vocational education. The model indicates relations between the team's beliefs about the interpersonal context, team learning behaviours and team effectiveness. Here, team entitativity was included as it shows considerable overlap with the included beliefs. Data were collected from 105 teams (total $\mathrm{N}=488$ members) and analysed using three-step multilevel analysis that showed that the suggested research model applied to our data. 